Original Research Paper

\title{
Two Co-Expression Strategies for Eicosapentaenoic Acid Production in Mortierella alpina
}

\author{
1, 2Xin Tang, 1, 2Haiqin Chen, ${ }^{1,2}$ Xiaoke Zhang, ${ }^{1,2}$ Zhennan Gu, \\ 1, 2, 3, ${ }^{4}$ Hao Zhang, ${ }^{1,2,3,4}$ Yong Q. Chen and ${ }^{1,2,3,5}$ Wei Chen \\ ${ }^{1}$ State Key Laboratory of Food Science and Technology, Jiangnan University, Wuxi, Jiangsu 214122, China \\ ${ }^{2}$ School of Food Science and Technology, Jiangnan University, Wuxi, Jiangsu 214122, China \\ ${ }^{3}$ National Engineering Research Center for Functional Food, Jiangnan University, Wuxi, Jiangsu 214122, China \\ ${ }^{4}$ Wuxi Translational Medicine Research Center and Jiangsu Translational Medicine Research Institute Wuxi Branch, \\ Wuxi, Jiangsu 214122, China \\ ${ }^{5}$ Beijing Innovation Centre of Food Nutrition and Human Health, \\ Beijing Technology and Business University (BTBU), Beijing 100048, China
}

Article history

Received: 05-08-2019

Revised: 06-10-2019

Accepted: 04-12-2019

Corresponding Author:

Haiqin Chen

State Key Laboratory of Food Science and Technology, Jiangnan University, Wuxi, Jiangsu 214122, China Tel: +8651085197239 Email: haiqinchen@jiangnan.edu.cn

\begin{abstract}
The omega-3 long-chain Polyunsaturated Fatty Acid (PUFA) Eicosapentaenoic Acid (EPA) has beneficial effects on human health, leading to its use in health products and foods. The oleaginous microorganism Mortierella alpina has been used in the industrial production of Arachidonic Acid (AA). The omega-3 desaturase oPpFADS17 is a key enzyme in the bioconversion of AA to EPA from Phytophthora parasitica and Glucose-6-Phosphate Dehydrogenase (G6PD2) is a critical enzyme that provide reducing power NADPH for lipid biosynthesis. In this study, we used a double expression cassette and $2 \mathrm{~A}$ peptide strategies to co-express the these two critical enzymes. Subsequently, we investigated the effects of these strategies on total lipid and EPA accumulation. The recombination strains generated using the above-described strategies exhibited no differences in cell growth, compared with the control strain. Recombination strains generated using the double expression cassette exhibited an increase in total lipids to $43 \%$ of the cell dry weight, but did not accumulate EPA. Recombination strains generated using the $2 \mathrm{~A}$ peptide strategy exhibited increased EPA accumulation, such that this PUFA accounted for $30 \%$ of the total lipid content. These co-expression strategies provide the improvements of multigene modification in PUFA accumulation in M. alpina.
\end{abstract}

Keywords: Co-expression, Double Expression Cassette, 2A Peptide, Mortierella alpine, EPA

\section{Introduction}

Eicosapentaenoic Acid (EPA, C20:5), an omega-3 long-chain Polyunsaturated Fatty Acid (LC-PUFA), has attracted considerable attention due to its beneficial effects on human health, including the prevention and treatment of diabetes, immune disorders, cardiovascular disease and cancer (Hirahashi et al., 2014; Maehre et al., 2015; Xue et al., 2013). Within the food chain, EPA is mainly accumulated in marine fish. However, the market demand for this food source exceeds the supply because of human population growth, environmental pollution and limitations associated with the harvest season and location (Ji et al., 2015; Tang et al., 2018; Xie et al., 2017).
Various microorganisms, including Mortierella alpina and Yarrowia lipolytica, have been investigated as potential hosts for the sustainable commercial production of EPA (Ando et al., 2009; Xue et al., 2013). M. alpina, an oleaginous filamentous fungus currently used to produce commercial Arachidonic Acid (AA, C20:4), can generate EPA from AA via an omega-3 desaturase (EC 1.14.19.-) (Ge et al., 2017; Ji et al., 2014; Okuda et al., 2015). Our latest research identified a new omega-3 desaturase, oPpFADS17, from Phytophthora parasitica. This enzyme converts AA to EPA at a conversion rate of $70 \%$. When expressed in a recombinant strain of $M$. alpina, oPpFADS17 increased the proportion of EPA among the Total Fatty Acids (TFA) content to $31.5 \%$ while the control 
strain without the recombinant enzyme didn't accumulate EPA (Tang et al., 2018). Although this recombinant strain represents a breakthrough in EPA production, further enhancement of the TFA content could potentially increase the EPA yield further. Another previous study demonstrated that the Pentose Phosphate Pathway (PPP), especially Glucose-6-Phosphate Dehydrogenase (G6PD2, EC1.1.1.49), plays a major role in the accumulation of total lipids (Hao et al., 2016). Accordingly, the co-expression of oPpFADS17 and G6PD2 may represent a good strategy toward improving EPA production in M. alpina.

The double expression cassette strategy can be used to co-express multiple copies of a gene or multiple genes with independent promoters (Bouabe et al., 2008). Another multi-gene construction strategy, the selfsplicing 2A peptide, is one of the most widely used tools for the construction of polygenic vector and has also attracted extensive attention (Park et al., 2009; Szymczak et al., 2004). The 2A peptide strategy exhibits obvious advantages with respect to protein activity and the expression of multiple downstream genes. Accordingly, it appears to be an ideal gene expression strategy (Hu et al., 2009; Trichas et al., 2008). Previous studies revealed that $2 \mathrm{~A}$ peptide could be applied successfully in a broad range of organisms, including fungi (de Felipe et al., 2003), plants (Halpin et al., 1999; Park et al., 2009), mammalian cells (Hu et al., 2009; Szymczak et al., 2004) and transgenic animals (Trichas et al., 2008). In this study, we cooverexpressed oPpFADS17 and G6PD2 using both the double expression cassette and 2A peptide strategies with the aim of improving EPA production in $M$. alpina.

\section{Materials and Methods}

\section{Strains and Culture Media}

M. alpina strains were maintained on $\mathrm{GY}$ medium, which contained $0.3 \mathrm{~g} / \mathrm{L} \mathrm{MgSO} 4 \cdot 7 \mathrm{H}_{2} \mathrm{O}, 1 \mathrm{~g} / \mathrm{L} \mathrm{NaH}_{2} \mathrm{PO}_{4}, 2$ $\mathrm{g} / \mathrm{L} \mathrm{KNO}_{3}, 5 \mathrm{~g} / \mathrm{L}$ yeast extract and $30 \mathrm{~g} / \mathrm{L}$ glucose. Uracil $(0.05 \mathrm{mg} / \mathrm{mL})$ and 5-fluoroorotic acid $(5-F O A, 0.5 \mathrm{mg} / \mathrm{mL})$ were added to the medium when culturing uracil auxotrophs. Agrobacterium tumefaciens AGL-1 was cultured in YEP medium, which contained $5 \mathrm{~g} / \mathrm{L} \mathrm{NaCl}, 10$ $\mathrm{g} / \mathrm{L}$ yeast extract and $10 \mathrm{~g} / \mathrm{L}$ tryptone. Synthetic Complete (SC) medium, Minimal Medium (MM) and Induction Medium (IM) were described previously (Hao et al., 2014).

\section{Growth Conditions}

Escherichia coli DH5 $\alpha$ was cultivated at $37^{\circ} \mathrm{C}$ on LB agar medium containing $10 \mathrm{~g} / \mathrm{L} \mathrm{NaCl}, 10 \mathrm{~g} / \mathrm{L}$ tryptone and $5 \mathrm{~g} / \mathrm{L}$ yeast extract. A. tumefaciens AGL-1 was grown at $28^{\circ} \mathrm{C}$ in YEP agar medium. M. alpina strains were cultured at $28^{\circ} \mathrm{C}$ in broth medium at $200 \mathrm{rpm}$ for $168 \mathrm{~h}$ (Wang et al., 2018).
Construction of the Transfer DNA Binary Vector Using the Double Expression Cassette Strategy

The pBIG2-ura5s-G6PD2 plasmid was extracted (Hao et al., 2016) and the entire G6PD2 expression unit (including the promoter and terminator) was obtained using Polymerase Chain Reaction (PCR) with G6PD2F1/R1 primer sequences. The underlined regions of the sequences indicate the XbaI restriction site (Table 1). Subsequently, the purified G6PD2 expression unit fragment and the extracted plasmid expression vector pBIG2-ura5s-oPpFADS17 (Tang et al., 2018) were digested using the restriction endonuclease XbaI. Recombinant ligase (Exnase) was then used to construct the binary expression vector pBIG2-ura5s-oPpFADS17G6PD2 using one step cloning kit (Takara).

Construction of the Transfer DNA Binary Vector Using the 2A Peptide Strategy

The 2A sequences are listed in Table 1 (produced by GenScrip.China). The target gene was digested with HindIII/XmaI and ligated into the binary vector pBIG2ura5s-Its (Chen et al., 2015). According to the 2A peptide co-overexpression strategy, oPpFADS17 and G6PD2 were amplified using the oPpFADS17-F/R and G6PD2-F2/R2 primer pairs, respectively. First, $o P p F A D S 17$ and pBIG2-ura5s-2A were separately digested with HindIII and NheI and then ligated to construct pBIG2-ura5s-oPpFADS17-2A. G6PD2 and pBIG2-ura5s-oPpFADS17-2A were then digested using $K p n I$ and XmaI and ligated together to generate pBIG2ura5s-oPpFADS17-2A-G6PD2.

\section{A. Tumefaciens-Mediated Transformation (ATMT)}

ATMT was performed as described previously (Hao et al., 2014). Briefly, M. alpina uracil-auxotrophic strain spores were harvested and diluted with fresh liquid GY medium to a concentration of $10^{8}$ spores $/ \mathrm{mL}$. A. tumefaciens AGL-1 was electro-transformed at $2.5 \mathrm{KV}$ and $5.0 \mathrm{~ms}$, then the transformants were cultured and diluted to an $\mathrm{OD}_{600}$ of 0.3 with fresh IM. Subsequently, the cells were incubated to an $\mathrm{OD}_{600}$ of 0.9 , mixed with spore suspension and spread onto on solid IM with uracil of $0.05 \mathrm{~g} / \mathrm{L}$ and transferred onto uracil-free SC medium with $100 \mu \mathrm{g} / \mathrm{mL}$ cefotaxime and $100 \mu \mathrm{g} / \mathrm{mL}$ spectinomycin. The obtained transformants were sub-cultured on uracil-free $\mathrm{SC}$ agar in three times to confirm the stability.

\section{Genomic DNA Preparation}

M. alpina strains were cultivated in liquid GY medium and the collected, then washed twice with sterile water. After removing most of the water, the mycelia were snap-frozen in liquid nitrogen. The DNA of $M$. alpina genomic was extracted as described previously (Wang et al., 2011). 
Table 1: Primers used in this study

\begin{tabular}{|c|c|}
\hline Primer & Sequence $\left(5^{\prime}-3^{\prime}\right) \mathrm{a}$ \\
\hline G6PD2-F1 for double & AGGTACACTTGTTTAGAGGTCTAGAT \\
\hline expression cassette strategy & TTAGTTGATGTGAGAGTTGTGAGATTCG \\
\hline G6PD2-R1 for double & AGGGAGTCACGTTATGACCTCTAGAC \\
\hline expression cassette strategy & CTCTAAACAAGTGTACCTGTGCATTCTG \\
\hline$o P p F A D S 17-\mathrm{F}$ for $2 \mathrm{~A}$ peptide strategy & ATCTTGAAGCTTCAATGGCTACCAAGCAGGCC \\
\hline$o P p F A D S 17-\mathrm{R}$ for $2 \mathrm{~A}$ peptide strategy & TCTAGA $\overline{\text { GCTAGCGGTGGACTTGGTCTTGACAG }}$ \\
\hline G6PD2-F2 for 2A peptide strategy & GCACGGGGTACCATGTCTGAGAAGAAGAAGCATCTTT \\
\hline G6PD2-R2 for 2A peptide strategy & GCTCCCㄷCCGGGTTAATGGTCAGTCCTTGTGTCCT \\
\hline \multirow[t]{9}{*}{$2 \mathrm{~A}$} & СТCTCCTATGAGTCGTTTACCCAGAATGCACAGGTACAㅅAGCTTTCCCA \\
\hline & AGCGAATTTGTCATCTCGACTGGTGCAAACTGCGCAAACGGCTGACTCA \\
\hline & TTGCCCA TGCTTTTCTTCTCCACGCCTATCGTCTGTTACTGCATCTCTGTC \\
\hline & GTGTTGAATGCGTAACTGATGGACTTCCGTATGGCTAGCGGCTCGGGAG \\
\hline & CCACCAACTTCTCCCTGCTCAAGCAGGCAGGAGACGTCGAGGAGAAC \\
\hline & CCCG GACCTGGTACCATGTCTGAGAAGAAGAAGCATCTTTACACTGTG \\
\hline & GTTGTGCTCGGTGCATCGGGAGACCTAGCCAAGAAGAAAACCTTCCCG \\
\hline & GCGCTCTTTGGACTCTTCAAAAACCAATATCTGGATCAAAACACGCA \\
\hline & CATC GTCGGCCCCGGG \\
\hline Hispro-F & CACACACAAАCСТСТСТСССАСТ \\
\hline TrpC-R & CAAATGAACGTATCTTATCGAGATCC \\
\hline$o P p F A D S 17-\mathrm{RTF}$ & TCTTCCCCACCCTCACCG \\
\hline$o P p F A D S 17-\mathrm{RTR}$ & CAAGCCACGAGCGTAGTTCA \\
\hline G6PD2-RTF & GCGTACAAAGATGGATCGG \\
\hline G6PD2-RTR & TGAAAGCCGTCGTCTGTG \\
\hline 18S-RTF & CGTACTACCGATTGAATGGCTTAG \\
\hline 18S-RTR & CCTACGGAAACCTTGTTACGACT \\
\hline
\end{tabular}

Reverse Transcriptase Quantitative PCR (RTqPCR) Analysis

RT-qPCR was carried out as described previously (Chen et al., 2015). Total RNA was extracted from the $M$. alpina using TRIzol reagent and reverse transcribed by the PrimeScript RT reagent kit. The primer pairs used for RTqPCR were shown in Table 1. RT-qPCR was performed using the ABI Prism 7900 sequence detection system and Power SYBR green PCR master mix. The expression levels of all genes were normalised to the expression of the internal control gene (18S rRNA). The date were quantified by the method of $2^{-\Delta \Delta C t}$.

\section{Determination of Enzymatic Activity}

Mycelia were collected by filtration, snap-frozen, ground in liquid nitrogen and suspended in extraction buffer (Chen et al., 2015). The suspension was centrifuged and collected the supernatant, then the protein concentration was determined by the Bradford method. The enzymatic activities of G6PD were detected as described previously (Tang et al., 2015; 2014) and indicated by the NADPH production of per min and per mg protein.

\section{NADPH Quantification}

The $M$. alpina mycelia were rapidly collected, frozen and ground in liquid nitrogen. The NADPH levels were determined using a NADPH quantification colorimetric kit (Biovision, Milpitas, CA, USA) according to the manufacturers' instruction.

Cell Dry Weight (CDW) and Fatty Acid Methyl Esters (FAME) Analysis

Fungal mycelia were harvested, washed twice with distilled water and frozen in liquid nitrogen. After lyophilisation, the CDW was determined gravimetrically. Approximately $20 \mathrm{mg}$ (dry weight) of mycelia were subjected to fatty acid extraction and methyl esterification. The profiles of FAMEs were analysed using gas chromatography-mass spectrometry as previously (Chen et al., 2015).

Statistical Analysis

The experiments were carried out in three biological replicates and the mean values and standard errors were calculated. A statistical analysis of the data was performed by SPSS 20.0. One-way analysis of variance (ANOVA) was conducted on the data and $P<0.05$ was considered significantly different.

\section{Results}

Co-expression oPpFADS17 and G6PD2 in $M$. Alpina Using the Double Expression Cassette and $2 A$ Peptide Strategies

To generate a high-EPA-producing strain, oPpFADS17 and G6PD2 were amplified and co- 
overexpressed in $M$. alpina using the double expression cassette strategy. The recombinant plasmid pBIG2ura5s-oPpFADS17-2A-G6PD2 was constructed as shown in Fig. 1. The recombinant vectors yielded target gene fragments of 818, 1256 and $1711 \mathrm{bp}$, indicating that the double expression cassette strategy was used to successfully construct the co-expression vector pBIG2ura5s-oPpFADS17-G6PD2.

To compare the effectiveness of co-expression between the two strategies, oPpFADS17 and G6PD2 were also amplified and co-overexpressed in $M$. alpina using the $2 \mathrm{~A}$ peptide strategy (Fig. 2). The recombinant vector $\mathrm{pBIG} 2$-ura5s-oPpFADS17-G6PD2 yielded target gene fragments of 818 and $2879 \mathrm{bp}$, indicating the successful construction of the coexpression vector.

The double expression cassette- and 2A peptidegenerated recombinant plasmids were transformed into M. alpina using ATMT. Six transformants each were generated using the double expression cassette (MA-oPpFADS17-G6PD2-1/2/3/4/5/6) and 2A peptide strategies (MA-oPpFADS17-2A-G6PD21/2/3/4/5/6) (Fig. 3). The double expression cassette transformants yielded three fragments $(818,1256$ and $1711 \mathrm{bp}$ ), whereas the wild-type control strain did not yield these fragments. These data indicated successful insertion of the cassette into the genome (Fig. 3A). The $2 \mathrm{~A}$ peptide transformants were shown to harbour the $u r a 5$ and oPpFADS17-2A-G6PD2 expression cassettes, as indicated by the presence of two inserted T-DNA fragments which of 818 and 2879 bp (Fig. 3B). In other words, both cassettes were successfully inserted into the genomes of the transformants.

Cell Growth and Lipid Accumulation in oPpFADS17 and G6PD2 Co-Expressing Strains

Next, the CDW, TFA content and EPA content of each $M$. alpina recombination strain was measured to investigate the effects of oPpFADS17 and G6PD2 coexpression via both strategies on cell growth and lipid accumulation (Fig. 4 and 5). As shown in Fig. 4A and $5 \mathrm{~A}$, neither recombination strategy appeared to have a significant effect on CDW when the recombinant strains were compared with the control strain $M$. alpina ATCC 32222. In other words, the inserted genes did not affect cell growth. However, the recombination strains generated using the double expression cassette strategy exhibited clear increases in the TFA and MA-oPpFADS17-G6PD2-1/2 contents to $42.2 \%$ and $43.0 \%$ (the value of control strain is $34.9 \%$ ), respectively, whereas the strains generated using the $2 \mathrm{~A}$ peptide strategy did not differ from the control strain (Fig. 4B). Moreover, EPA was not detected in any of the recombination strains generated using the double expression cassette strategy (Fig. $4 C)$. In other words, the co-expression of oPpFADS 17 and G6PD2 via a double expression cassette increased the TFA but had no effect on EPA accumulation. In contrast, the TFA contents did not differ significantly between the recombination strains generated using the 2A peptide strategy and the control strain (Fig. 5B). Meanwhile, the recombination strains generated using the $2 \mathrm{~A}$ peptide strategy varied widely with respect to the ratio of EPA to the TFA content. Specifically, the EPA contents in the strains MA-oPpFADS17-2AG6PD2-1/2/3/4 increased to $29.4 \%, 30.6 \%, 2.4 \%$ and $4.1 \%$ (EPA is not detected in control strain), respectively, whereas EPA was not detected in MA-oPpFADS17-2AG6PD2-5/6 (Fig. 5C). The differences in the TFA and EPA contents of these $2 \mathrm{~A}$ peptide-generated recombination strains can be attributed to random integration based on the ATMT method (Ando et al., 2009).
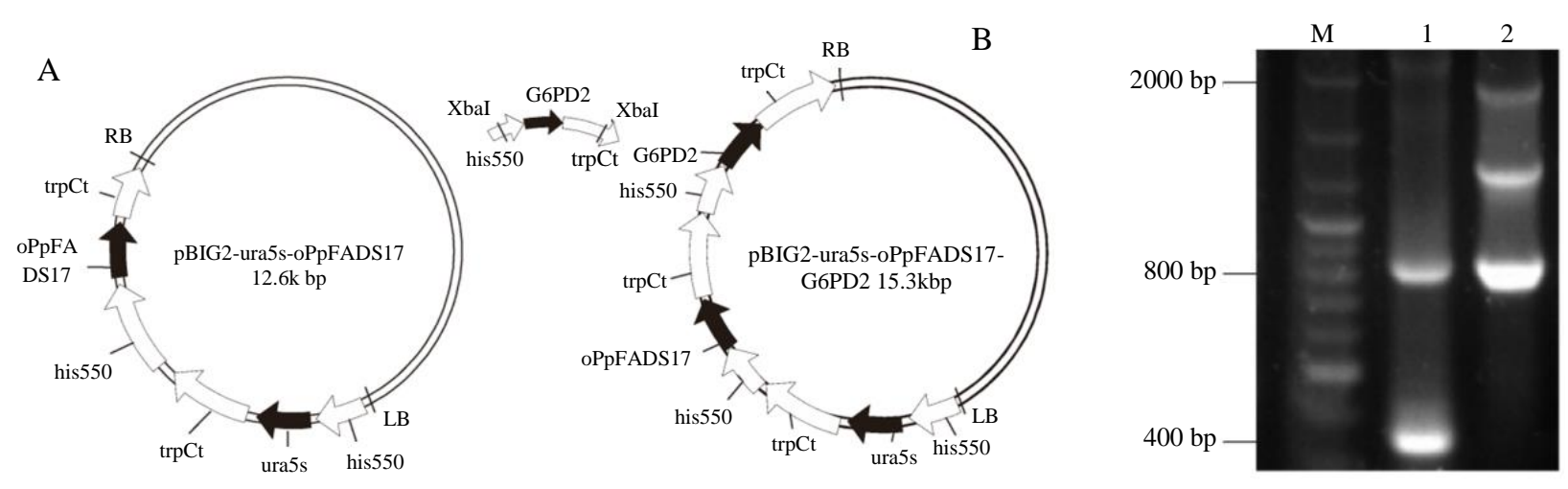

Fig. 1: Construction of the binary vector pBIG2-ura5s-oPpFADS17-G6PD2 using double expression cassette strategy (A) and PCR verification of the recombinant plasmid (B). M, Marker; 1, pBIG2-ura5s-ITs; 2, pBIG2-ura5s-oPpFADS17-G6PD2 

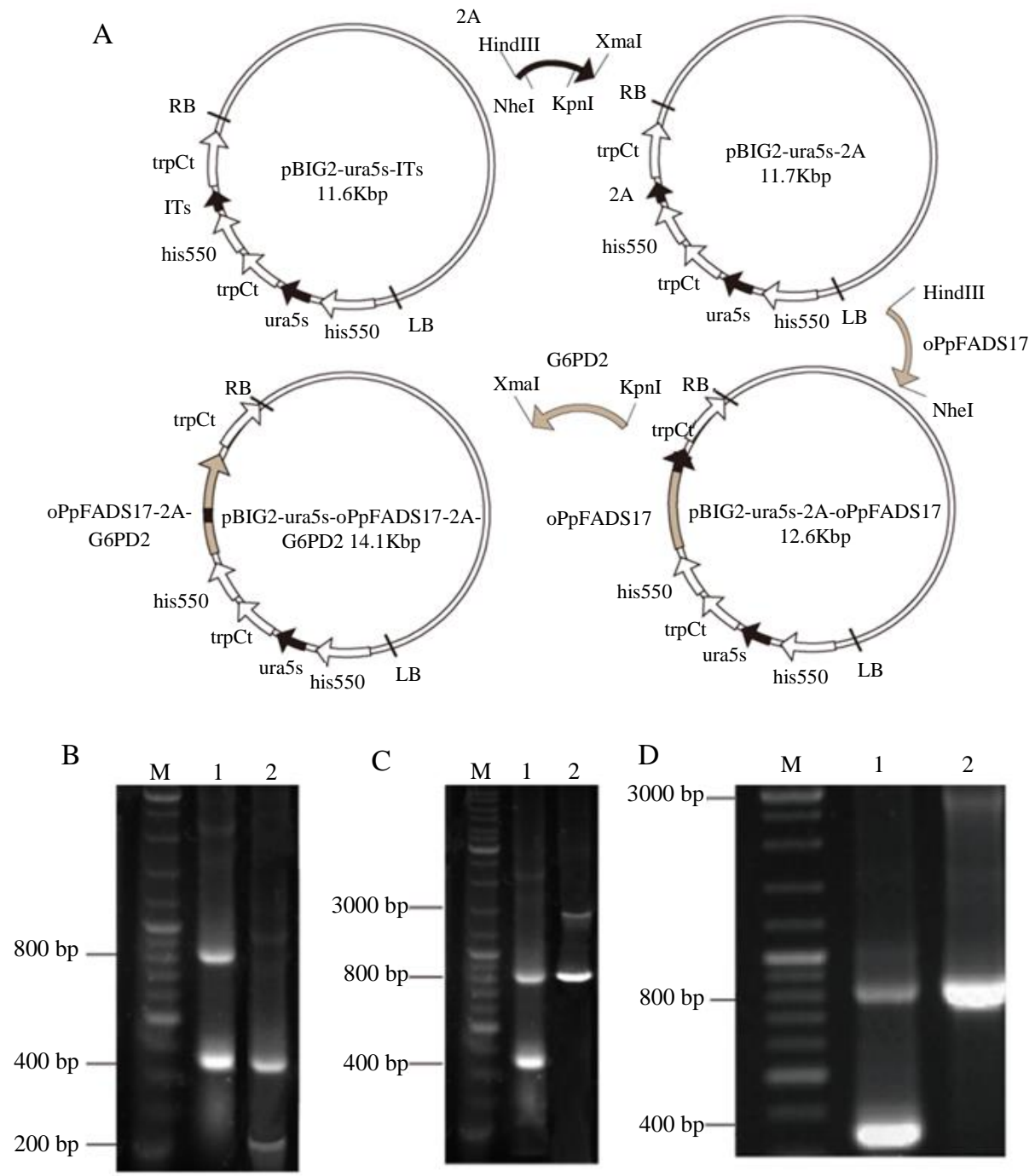

Fig. 2: Construction of the binary vector pBIG2-ura5s-oPpFADS17-2A-G6PD2 using 2A peptide strategy (A) and PCR verification of the recombinant plasmid [M, Marker; 1, pBIG2-ura5s-Its; 2, pBIG2-ura5s-2A (B)/pBIG2-ura5s-oPpFADS17-2A (C)/pBIG2-ura5s-oPpFADS17-2A-G6PD2 (D)]
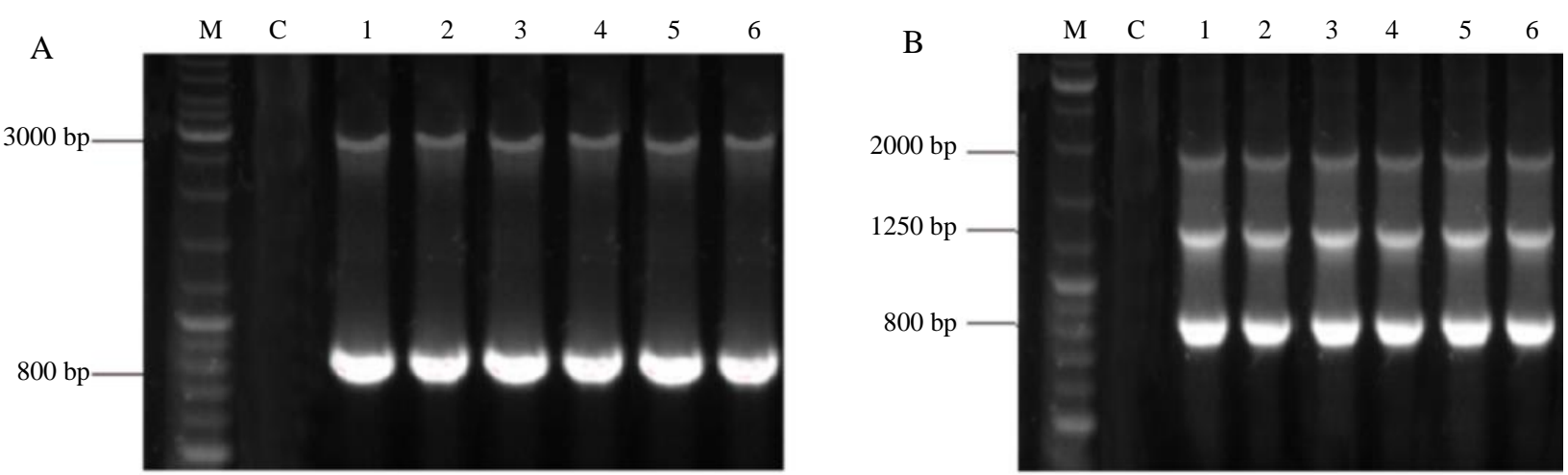

Fig. 3: PCR verification of the transformants. The presence of integrated $o P p F A D S 17$ and G6PD2 was verified by PCR with the primer pair Hispro-F/TrpC-R. Lane M, marker; lane C, M. alpina ATCC 32222 (negative control); lanes 1/2/3/4/5/6, MAoPpFADS17-G6PD2-1/2/3/4/5/6 using double expression cassette strategy (A), MA-oPpFADS17-2A-G6PD2-1/2/3/4/5/6 using $2 \mathrm{~A}$ peptide strategy $(\mathrm{B})$ 

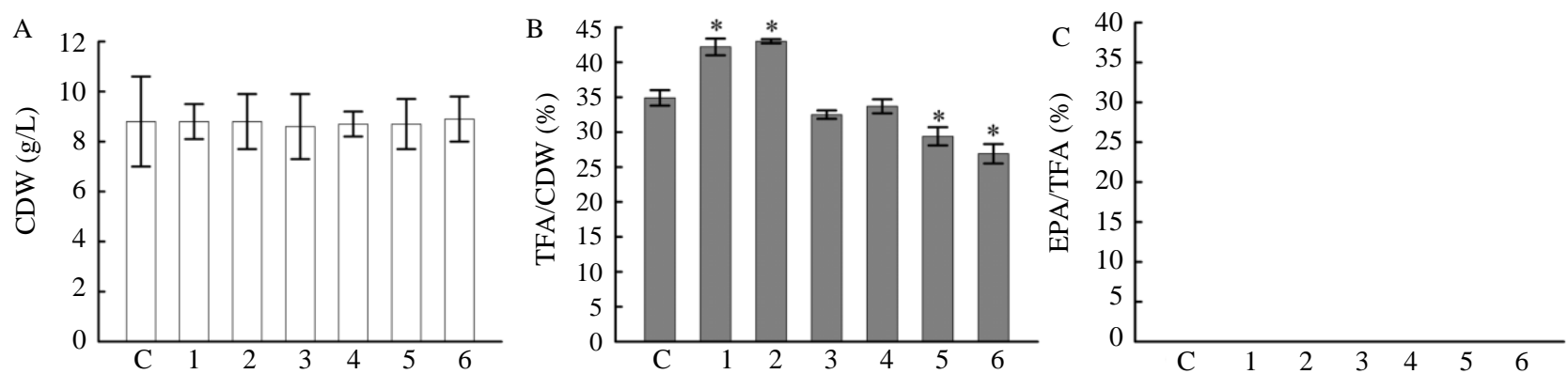

Fig. 4: Cell dry weight (CDW), total fatty acids (TFA) content (w/w, TFA/CDW) and EPA content (w/w, EPA/TFA) in $o P p F A D S 17$ and G6PD2 co-expressing strains generated using the double expression cassette strategy. C, M. alpina ATCC 32222 (negative control), 1/2/3/4/5/6, MA-oPpFADS17-G6PD2-1/2/3/4/5/6. Strains were cultured at $28{ }^{\circ} \mathrm{C}$ in $250-\mathrm{mL}$ shaking flasks containing $100 \mathrm{~mL}$ of broth medium at $200 \mathrm{rpm}$ for $168 \mathrm{~h}$. Three independent experiments were performed and the error bars represent the standard deviations. $* P<0.05$ indicates a significant difference relative to the $\mathrm{C}$ group
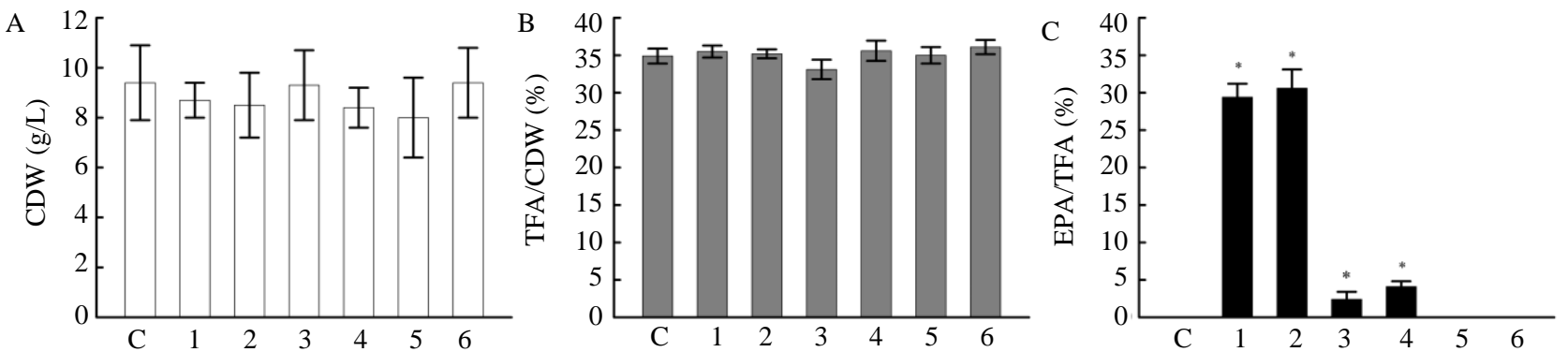

Fig. 5: Cell dry weight (CDW), total fatty acids (TFA) content (w/w, TFA/CDW) and EPA content (w/w, EPA/TFA) in oPpFADS17 and G6PD2 co-expressing strains generated using the 2A peptide strategy. C, M. alpina ATCC 32222 (negative control), 1/2/3/4/5/6, MA-oPpFADS17-2A-G6PD2-1/2/3/4/5/6. Strains were cultured at $28^{\circ} \mathrm{C}$ in 250 -mL shaking flasks containing $100 \mathrm{~mL}$ of broth medium at $200 \mathrm{rpm}$ for $168 \mathrm{~h}$. Three independent experiments were performed and the error bars represent standard deviations. ${ }^{*} P<0.05$ indicates a significant difference relative to the $\mathrm{C}$ group

\section{Expression Levels of the oPpFADS17 and G6PD2 in M. Alpina}

The transcription levels of $o P p F A D S 17$ and G6PD2 in the MA-oPpFADS17-G6PD2-1/2 (double expression cassette strategy) and MA-oPpFADS17-2A-G6PD2-1/2 strains (2A peptide strategy) grown in broth liquid medium for $168 \mathrm{~h}$ were determined using RT-qPCR (Fig. 6). As wild-type M. alpina does not express $O P p F A D S 17$, we used a single gene-overexpressing strain (MA-oPpFADS17) from the previous study (Tang et al., 2018) as the control strain (Fig. 6A). In the transformants generated using the double expression cassette strategy, the respective level of oPpFADS17 transcription in MAoPpFADS17-G6PD2-1 and -2 either did not differ significantly or was $16 \%$ lower when compared with the control strain. In the transformants using the $2 \mathrm{~A}$ peptide strategy, the respective level of oPpFADS17 transcription in MA-oPpFADS17-2A-G6PD2-1 and -2 either did not differ significantly or increased by $26 \%$ when compared with the control strain. The results showed that the transcription level of the $o P p F A D S 17$ occurred in these transformants. The levels of G6PD2 transcription in the MA-oPpFADS17-G6PD2-1/2 transformants were approximately five- and three-fold higher than that in the wild-type strain M. alpina ATCC 32222. However, the levels of G6PD2 transcription in MAoPpFADS17-2A-G6PD2-1/2 did not increase relative to that of the wild-type strain (Fig. 6B). These results suggest that the double expression cassette strategy led to the successful insertion and transcription of G6PD2, whereas the $2 \mathrm{~A}$ peptide strategy had no significant effect on G6PD2 transcription.

To investigate whether the enzyme encoded by G6PD2 retained its activity in the transformants generated using the two strategies, the activity of G6PD and the production of NADPH, a product of catalysis supplied directly for lipid synthesis, were determined (Fig. 7). The levels of G6PD activity and NADPH in the transformants generated using the double expression cassette strategy increased significantly when compared with the wild-type strain. In contrast, neither level was affected in the transformants generated using the $2 \mathrm{~A}$ peptide strategy, compared with the wild-type strain. These results were consistent with the transcription level data. 

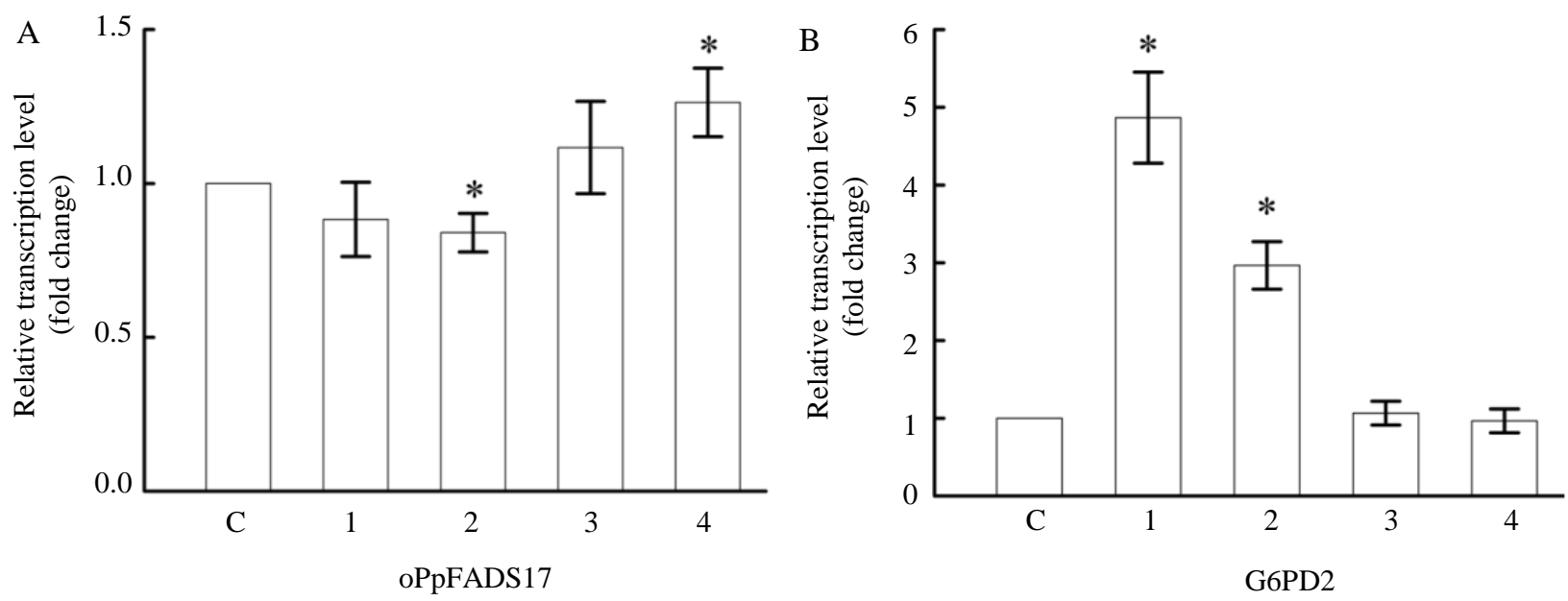

Fig. 6: Relative levels of $o P p F A D S 17$ and G6PD2 transcription in M. alpina transformants. C, MA-oPpFADS17 (A)/M. alpina ATCC 32222 (B); 1, MA-oPpFADS17-G6PD2-1 using double expression cassette strategy; 2, MA-oPpFADS17-G6PD2-2 using double expression cassette strategy; 3, MA-oPpFADS17-2A-G6PD2-1 using 2A peptide strategy; 4, MA-oPpFADS17$2 A-G 6 P D 2-2$ using $2 \mathrm{~A}$ peptide strategy. Strains were cultured at $28^{\circ} \mathrm{C}$ in $250-\mathrm{mL}$ shaking flasks containing $100 \mathrm{~mL}$ of broth medium at $200 \mathrm{rpm}$ for $168 \mathrm{~h}$. Three independent experiments were performed. Error bars represent standard deviations. * indicates a significant difference from the $\mathrm{C}$ group $(P<0.05)$
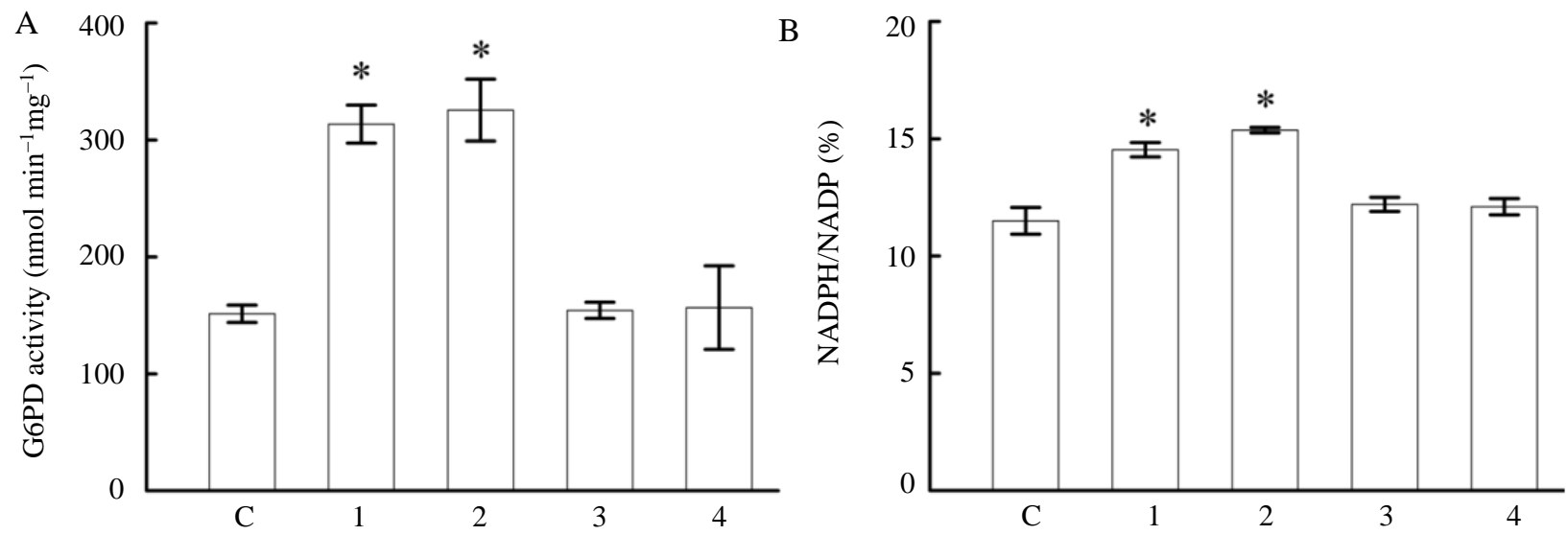

Fig. 7: The G6PD activity (A) and NADPH levels (B) in M. alpina transformants. C, M. alpina ATCC 32222; 1, MA-oPpFADS17G6PD2-1 using double expression cassette strategy; 2, MA-oPpFADS17-G6PD2-2 using double expression cassette strategy; 3, MA-oPpFADS17-2A-G6PD2-1 using 2A peptide strategy; 4, MA-oPpFADS17-2A-G6PD2-2 using 2A peptide strategy. The strains were cultured at $28^{\circ} \mathrm{C}$ in 250 - $\mathrm{mL}$ shaking flasks containing $100 \mathrm{~mL}$ of broth medium at $200 \mathrm{rpm}$ for $168 \mathrm{~h}$. Three independent experiments were performed and the error bars represent standard deviations. * indicates a significant difference when compared with the $\mathrm{C}$ group $(P<0.05)$

\section{Discussion}

The omega-3 desaturase oPpFADS17 is the key enzyme in the bioconversion of AA to EPA and G6PD2 can provide reducing power NADPH for lipid biosynthesis (Hao et al., 2016; Tang et al., 2017; 2018). In this study, we coexpressed oPpFADS17 and G6PD2 in M. alpina using both the double expression cassette and $2 \mathrm{~A}$ peptide strategies and studied the effects of co-expression on total lipid and EPA accumulation. In the transformants generated using the double expression cassette strategy, the total lipid content increased but no accumulated EPA was detected. Possibly, the promoters in the double expression cassette strategy interfered with each other and affected the expression of the target gene. Additionally, excessively long target fragments may have increased the burden on the host cells and affected cell metabolism. Several studies showed that the target product expression level correlated with the copy number of the corresponding expression cassette (Fang et al., 2014; Mansur et al., 2005; Per et al., 2011; Wu et al., 2014; Zhu et al., 2009). According to Yu et al. (2003) 
different combinations of genes and promoters have important effects on promoter activity and high combinatorial matching could better achieve gene expression. In addition, the enhancer element of one transcription unit may activate the distal promoter of another unit, resulting in cross-interference between two adjacent expression units. Although insulators can be used to prevent this interference, the integration of primitive insulators with internal promoters/enhancers may also reduce the potential activities of adjacent genes. Consistent with this possibility, the first expression unit in our study did not achieve the desired effect and was likely controlled by the enhancer of another expression unit, resulting in abnormal expression.

In many organisms, the $2 \mathrm{~A}$ peptide facilitates the long-term stable co-expression of multiple genes (de Felipe et al., 2006). Indeed, we used the 2A peptide strategy to establish a feasible system for the coexpression of multiple genes in M. alpina. Here, the EPA content increased to as much as $30 \%$ of the TFA level and the level of $o P p F A D S 17$ transcription was higher than that in the wild-type strain. However, the TFA content did not increase significantly in transformants generated using this strategy, possibly due to the relatively low shear efficiency of the $2 \mathrm{~A}$ peptide in the recombinant strains. Previous studies indicated that different 2A peptide exhibited various shear efficiencies in different hosts (Donnelly et al., 2001; Doronina et al., 2008; Luke et al., 2008; Szymczak et al., 2004). The shear efficiency of the $2 \mathrm{~A}$ peptide may be associated with two problems. First, the shear efficiencies of polyproteins translated before and after the $2 \mathrm{~A}$ peptide may differ. Second, the fusion of $2 \mathrm{~A}$ peptide residues to the $\mathrm{C}$-terminal of the upstream translation protein may interfere with the upstream protein function. The shear activity of the $2 \mathrm{~A}$ peptide may be related to the total amount of precursor protein. Moreover, G6PD2 plays an important role in the pentose phosphate pathway and its transcription and enzyme activity levels are high. In the presence of a high total amount of precursor protein, the shear activity of the $2 \mathrm{~A}$ peptide may decrease and the recombinant bacteria would not achieve the expected increase in total lipids. The polycistron employed a single promoter to drive mRNA transcripts containing multi-coding areas and therefore the strength of promoter is very important for the expression of target mRNA. In the $2 \mathrm{~A}$ peptide strategy of this study, the promoter successfully drived the transcription of $o P p F A D S 17$ but may be unable to drive another G6PD2 co-expression gene. This observation is in accordance with other studies that showed the efficiency of heterologous expression and gene transformation were influenced by different promoter (Cheng et al., 2009; Kilaru et al.,
2006). Also, the mRNA stability is another important factor in gene expression (Hargrove and Schmidt, 1989). The longer mRNA fragments were more likely to experience endonucleolytic attacks by RNA endonucleases and/or mechanical damage (Feng and Niu, 2007; Trcek et al., 2011). A nearly 3000-bp length might exceed the capacity to maintain mRNA stability.

\section{Conclusion}

In this study, we used double expression cassette and 2A peptide strategies to co-express the omega-3 desaturase oPpFADS17 and G6PD2 in M. alpina and investigated the effects on the accumulation of total lipids and EPA. In summary, recombination strains generated using the double expression cassette strategy exhibited an increase in the total lipids but no change in EPA accumulation, whereas those generated using $2 \mathrm{~A}$ peptide exhibited an increase in EPA accumulation up to $30 \%$ of the total lipid content. These multiple gene coexpressing strategies are expected to further enhance the accumulation of PUFAs in M. alpina.

\section{Funding Information}

This research was supported by the National Natural Science Foundation of China (31901659, 31722041), the Fundamental Research Funds for the Central Universities (JUSRP51702A), the Project funded by China Postdoctoral Science Foundation (2017M611701), the Postdoctoral Science Foundation of Jiangsu Province (1701061C), the National First-class Discipline Program of Food Science and Technology (JUFSTR20180102) and the Jiangsu Province Collaborative Innovation Center for Food Safety and Quality Control.

\section{Author Contributions}

Xin Tang and Xiaoke Zhang: Carried out the experiments and drafted the manuscript.

Haiqin Chen and Zhennan Gu: Analyzed the data and helped to draft the manuscript.

Haiqin Chen, Hao Zhang, Yong Q. Chen and Wei Chen: Conceived and designed the study and revised the manuscript. All authors read and approved the final manuscript.

\section{Conflict of Interest}

The authors have no conflict of interest.

\section{Ethics Statement}

The article has no study within human participants or animals. 


\section{References}

Ando, A., Y. Sumida, H. Negoro, D.A. Suroto and J. Ogawa et al., 2009. Establishment of Agrobacterium tumefaciens-mediated transformation of an oleaginous fungus, Mortierella alpina $1 \mathrm{~S}-4$ and its application for eicosapentaenoic acid producer breeding. Applied En. Microbiol., 75: 5529-5535. DOI: 10.1128/AEM.00648-09

Bouabe, H., R. Fässler and J. Heesemann, 2008. Improvement of reporter activity by IRES-mediated polycistronic reporter system. Nucleic Acids Res., 36: e28-e28. DOI: 10.1093/nar/gkm1119

Chen, H., G. Hao, L. Wang, H. Wang and Z. Gu et al., 2015. Identification of a critical determinant that enables efficient fatty acid synthesis in oleaginous fungi. Scientific Reports, 5: 11247-11247.

DOI: $10.1038 /$ srep 11247

Cheng, S., P. Yang, L. Guo, J. Lin and N. Lou, 2009. Expression of multi-functional cellulase gene MFC in Coprinus cinereus under control of different basidiomycete promoters. Biores. Technol., 100: 4475-4480. DOI: 10.1016/j.biortech.2009.04.021

de Felipe, P., G.A. Luke, L.E. Hughes, D. Gani and C. Halpin et al., 2006. E unum pluribus: Multiple proteins from a self-processing polyprotein. Trends Biotechnol., 24: 68-75.

DOI: $10.1016 /$ j.tibtech.2005.12.006

de Felipe, P., L.E. Hughes, M.D. Ryan and J.D. Brown, 2003. Co-translational, intraribosomal cleavage of polypeptides by the foot-and-mouth disease virus 2A peptide. J. Biol. Chem., 278: 11441-11448.

DOI: $10.1074 /$ jbc.M211644200

Donnelly, M.L., L.E. Hughes, G. Luke, H. Mendoza and E. ten Dam et al., 2001. The cleavage' activities of foot-and-mouth disease virus $2 \mathrm{~A}$ site-directed mutants and naturally occurring '2A-like'sequences. J. General Virol., 82: 1027-1041.

DOI: 10.1099/0022-1317-82-5-1027

Doronina, V.A., C. Wu, P. de Felipe, M.S. Sachs and M.D. Ryan et al., 2008. Site-specific release of nascent chains from ribosomes at a sense codon. Molecular Cellular Biol., 28: 4227-4239. DOI: 10.1128/MCB.00421-08

Fang, Z., X. Li, D. Pan, L. Jiao and Z. Liu et al., 2014. Enhanced production of thermomyces lanuginosus lipase in Pichia pastoris via genetic and fermentation strategies. J. Indus. Microbiol. Biotechnol., 41: 1541-1551. DOI: 10.1007/s10295-014-1491-7

Feng, L. and D.K. Niu, 2007. Relationship between mRNA stability and length: an old question with a new twist. Biochem. Genet., 45: 131-137.

DOI: $10.1007 / \mathrm{s} 10528-006-9059-5$
Ge, C., H. Chen, T. Mei, X. Tang and L. Chang et al., 2017. Application of a $\omega-3$ desaturase with an arachidonic acid preference to eicosapentaenoic acid production in Mortierella alpina. Frontiers Bioeng. Biotechnol., 5: 89-89.

DOI: $10.3389 /$ fbioe.2017.00089

Halpin, C., S.E. Cooke, A. Barakate, A.E. Amrani and M.D. Ryan, 1999. Self-processing 2A-polyproteinsa system for co-ordinate expression of multiple proteins in transgenic plants. Plant J., 17: 453-459. DOI: 10.1046/j.1365-313X.1999.00394.X

Hao, G., H. Chen, L. Wang, Z. Gu and Y. Song et al., 2014. Role of malic enzyme during fatty acid synthesis in the oleaginous fungus Mortierella alpina. Applied En. Microbiol., 80: 2672-2678. DOI: 10.1128/AEM.00572-16

Hao, G., H. Chen, Z. Gu, H. Zhang and W. Chen et al., 2016. Metabolic engineering of Mortierella alpina for enhanced arachidonic acid production through the NADPH supplying strategy. Applied En. Microbiol.

Hargrove, J.L. and F.H. Schmidt, 1989. The role of mRNA and protein stability in gene expression. FASEB J., 3: 2360-2370.

DOI: $10.1128 / A E M .00140-14$

Hirahashi, J., K. Kawahata, M. Arita, R. Iwamoto and K. Hishikawa et al., 2014. Immunomodulation with eicosapentaenoic acid supports the treatment of autoimmune small-vessel vasculitis. Scientific Reports.

Hu, T., Q. Fu, P. Chen, K. Zhang and D. Guo, 2009. Generation of a stable mammalian cell line for simultaneous expression of multiple genes by using 2A peptide-based lentiviral vector. Biotechnol. Lett., 31: 353-353. DOI: 10.1007/s10529-008-9882-3

Ji, X.J., L.J. Ren and H. Huang, 2015. Omega-3 biotechnology: A green and sustainable process for omega-3 fatty acids production. Frontiers Bioeng. Biotechnol., 3: 158-158.

DOI: $10.3389 /$ fbioe.2015.00158

Ji, X.J., L.J. Ren, Z.K. Nie, H. Huang and P.K. Ouyang, 2014. Fungal arachidonic acid-rich oil: Research, development and industrialization. Critical Rev. Biotechnol., 34: 197-214.

DOI: $10.3109 / 07388551.2013 .778229$

Kilaru, S., P.J. Hoegger, A. Majcherczyk, C. Burns and K. Shishido et al., 2006. Expression of laccase gene lcc1 in Coprinopsis cinerea under control of various basidiomycetous promoters. Applied Microbiol. Biotechnol., 71: 200-210.

DOI: $10.1007 / \mathrm{s} 00253-005-0128-1$

Luke, G.A., P. de Felipe, A. Lukashev, S.E. Kallioinen and E.A. Bruno et al., 2008. Occurrence, function and evolutionary origins of '2A-like'sequences in virus genomes. J. General Virol., 89: 1036-1042. DOI: 10.1099/vir.0.83428-0 
Maehre, H.K., I.J. Jensen, E.O. Elvevoll and K.E. Eilertsen, 2015. $\omega-3$ Fatty acids and cardiovascular diseases: Effects, mechanisms and dietary relevance. Int. J. Molecular Sci., 16: 22636-22661.

DOI: $10.3390 /$ ijms160922636

Mansur, M., C. Cabello, L. Hernández, J. País and L. Varas et al., 2005. Multiple gene copy number enhances insulin precursor secretion in the yeast Pichia pastoris. Biotechnol. Lett., 27: 339-345.

DOI: $10.1007 / \mathrm{s} 10529-005-1007-7$

Okuda, T., A. Ando, H. Negoro, T. Muratsubaki and H. Kikukawa et al., 2015. Eicosapentaenoic Acid (EPA) production by an oleaginous fungus Mortierella alpina expressing heterologous the $\Delta 17$-desaturase gene under ordinary temperature. Eur. J. Lipid Sci. Technol., 117: 1919-1927. DOI: 10.1002/ejlt.201400657

Park, S., K. Kang, Y.S. Kim and K. Back, 2009. Endosperm-specific expression of tyramine $\mathrm{N}$ hydroxycinnamoyltransferase and tyrosine decarboxylase from a single self-processing polypeptide produces high levels of tyramine derivatives in rice seeds. Biotechnol. Let., 31: 911-915. DOI: 10.1007/s10529-009-9951-2

Per, K., A. Erik, A.H. Danielson Jonas, A. Maria and N. Kristina et al., 2011. Increasing gene dosage greatly enhances recombinant expression of aquaporins in Pichia pastoris. BMC Biotechnol., 11: 47-47.

DOI: 10.1186/1472-6750-11-47

Szymczak, A.L., C.J. Workman, Y. Wang, K.M. Vignali and S. Dilioglou et al., 2004. Correction of multigene deficiency in vivo using a single'selfcleaving'2A peptide-based retroviral vector. Nature Biotechnol., 22: 589-589. DOI: 10.1038/nbt957

Tang, X., H. Chen, T. Mei, C. Ge and Z. Gu et al., 2018. Characterization of an omega-3 desaturase from Phytophthora parasitica and application for eicosapentaenoic acid production in Mortierella alpina. Frontiers Microbiol.

Tang, X., H. Chen, Y.Q. Chen, W. Chen and V. Garre et al., 2015. Comparison of biochemical activities between high and low lipid-producing strains of Mucor circinelloides: An explanation for the high oleaginicity of strain WJ11. PloS One, 10: e0128396-e0128396.

DOI: 10.1371/journal.pone.0128396

Tang, X., H. Chen, Z. Gu, H. Zhang and Y.Q. Chen et al., 2017. Comparative proteome analysis between high lipid-producing strain Mucor circinelloides WJ11 and low lipid-producing strain CBS 277.49. J. Agri. Food Chem., 65: 5074-5082.

DOI: $10.1021 /$ acs.jafc.7b00935
Tang, X., H. Zhang, H. Chen, Y.Q. Chen and W. Chen et al., 2014. Effects of 20 standard amino acids on the growth, total fatty acids production and $\gamma$ linolenic acid yield in Mucor circinelloides. Current Microbiol., 69: 899-908.

DOI: 10.1007/s00284-014-0671-z

Trcek, T., D.R. Larson, A. Moldón, C.C. Query and R.H. Singer, 2011. Single-molecule mRNA decay measurements reveal promoter-regulated mRNA stability in yeast. Cell, 147: 1484-1497. DOI: 10.1016/j.cell.2011.11.051

Trichas, G., J. Begbie and S. Srinivas, 2008. Use of the viral $2 \mathrm{~A}$ peptide for bicistronic expression in transgenic mice. BMC Biol., 6: 40-40.

DOI: $10.1186 / 1741-7007-6-40$

Wang, L., W. Chen, Y. Feng, Y. Ren and Z. Gu et al., 2011. Genome characterization of the oleaginous fungus Mortierella alpina. PloS One, 6: e28319-e28319. DOI: 10.1371/journal.pone.0028319

Wang, S., H. Chen, Y. Wang, X. Tang and H. Zhang et al., 2018. Optimization of Agrobacterium tumefaciensmediated transformation method of oleaginous filamentous fungus Mortierella alpina on cocultivation materials choice. J. Microbiol. Methods, 152: 179-185. DOI: 10.1016/j.mimet.2018.08.002

Wu, M., W. Liu, G. Yang, D. Yu and D. Lin et al., 2014. Engineering of a Pichia pastoris expression system for high-level secretion of HSA/GH fusion protein. Applied Biochem. Biotechnol., 172: 2400-2411. DOI: $10.1007 / \mathrm{s} 12010-013-0688-y$

Xie, D., E. Miller, P. Sharpe, E. Jackson and Q. Zhu, 2017. Omega-3 production by fermentation of Yarrowia lipolytica: From fed-batch to continuous. Biotechnol. Bioeng., 114: 798-812.

DOI: $10.1002 /$ bit.26216

Xue, Z., P.L. Sharpe, S.P. Hong, N.S. Yadav and D. Xie et al., 2013. Production of omega-3 eicosapentaenoic acid by metabolic engineering of Yarrowia lipolytica. Nature Biotechnol., 31: 734-740. DOI: $10.1038 /$ nbt.2622

Yu, X., X. Zhan, J. D'Costa, V.M. Tanavde and Z. Ye et al., 2003. Lentiviral vectors with two independent internal promoters transfer high-level expression of multiple transgenes to human hematopoietic stem-progenitor cells. Molecular Therapy J. Am. Society Gene Therapy, 7: 827-827.

DOI: $10.1016 / S 1525-0016(03) 00104-7$

Zhu, T., M. Guo, Z. Tang, M. Zhang and Y. Zhuang et al., 2009. Efficient generation of multi-copy strains for optimizing secretory expression of porcine insulin precursor in yeast Pichia pastoris. J. Applied Microbiol., 107: 954-954.

DOI: $10.1111 /$ j.1365-2672.2009.04279.x 